\title{
Study of the surface structure of butterfly wings using the scanning electron microscopic moiré method
}

\author{
Satoshi Kishimoto, ${ }^{1,2}$ Qinghua Wang, ${ }^{2}$ Huimin Xie, ${ }^{2, \star}$ and Yapu Zhao ${ }^{3}$ \\ ${ }^{1}$ Composites and Coatings Center, National Institute for Materials Science, Tsukuba, Ibaraki 305-0047, Japan \\ 2Department of Engineering Mechanics, FML, Tsinghua University, 100084 Beijing, China \\ ${ }^{3} \mathrm{LNM}$, Institute of Mechanics, Chinese Academy of Science, 100080 Beijing, China \\ ${ }^{*}$ Corresponding author: xiehm@mail.tsinghua.edu.cn
}

Received 18 April 2007; revised 24 July 2007; accepted 20 August 2007;

posted 20 August 2007 (Doc. ID 82184); published 27 September 2007

\begin{abstract}
Scanning electron microscopic (SEM) moiré method was used to study the surface structure of three kinds of butterfly wings: Papilio maackii Menetries, Euploea midamus (Linnaeus), and Stichophthalma howqua (Westwood). Gratings composed of curves with different orientations were found on scales. The planar characteristics of gratings and some other planar features of the surface structure of these wings were revealed, respectively, in terms of virtual strain. Experimental results demonstrate that SEM moiré method is a simple, nonlocal, economical, effective technique for determining which grating exists on one whole scale, measuring the dimension and the whole planar structural character of the grating on each scale, as well as characterizing the relationship between gratings on different scales of each butterfly wing. Thus, the SEM moiré method is a useful tool to assist with characterizing the structure of butterfly wings and explaining their excellent properties. (C) 2007 Optical Society of America
\end{abstract}

OCIS codes: $\quad 000.1430,050.2770,120.4120$.

\section{Introduction}

Butterfly wing has drawn great attention due to its excellent properties, such as attractive iridescence, superhydrophobic characteristics, and quick heat dissipation ability, which are closely related to its surface structure [1-3]. Butterfly wing is coated with many scales of $\sim 100-140 \mu \mathrm{m}$ length, arranged in an overlapping roof tile pattern. As a periodic structure, such as the regular array of atomic lattice or material bar, can be regarded as a grating, there is a grating formed by the approximately parallel longitudinal grooves and ridges on each scale and the orientations of these gratings are a little different. Among longitudinal ridges are many micro-cross-ribs, which can also form gratings with certain orientations. This special structure [4-6] can make incident light create some special optical effects such as multilayer interference $[7,8]$, diffraction $[9,10]$, scattering $[11]$, and photonic crystal effects [12], which lead to its attractive iridescence and also contribute a lot to its super-

0003-6935/07/287026-09\$15.00/0

(C) 2007 Optical Society of America hydrophobic characteristics [13] and quick heat dissipation ability. At present, the conventional observing and measuring method for the structure of butterfly wing uses high-power microscopies, such as scanning electron microscopy (SEM), transverse electron microscopy (TEM), scanning probe microscopy (SPM), scanning tunnel microscopy (STM), atomic force microscopy (AFM), etc. However, this method needs very high magnification for observation and is limited to a very small region each time, with the result that the whole structural character of the grating formed by the longitudinal ridges on one whole scale and the relationship between gratings on different scales of one butterfly wing are difficult to obtain through this point-by-point way. Besides, only the distribution of micro-cross-ribs in a very small region, which is not so much regular, can be seen in high-power microscopy, making it difficult to determine which grating existing on the whole scale on Earth, as various gratings with different orientations may be there just from visual sensation. Therefore, looking after a useful assistant for conventional methods, seeking a simple, nonlocal, economical, and effective technique for determining which grating ex- 
ists, measuring both the dimension and the whole planar structural character of the grating on each scale as well as characterizing the relationship between gratings on different scales of one butterfly wing, are of great importance.

Moiré method, using grating as the basic measuring element, has been proved to be a mature and effective optical metrology for in-plane deformation measurement and qualitative analysis of dislocation [14] for its high sensitivity and wide field of view, which is a prospective candidate for studying the surface planar structure of butterfly wing. Common moiré methods include geometric moiré method [15], moiré interferometry [16], electron beam moiré method [17], SEM moiré method [18], TEM moiré method [19] AFM moiré method [20], laser scanning confocal microscopy moiré method [21], etc. Since SEM possesses many advantages such as high resolution, long depth of field, wide magnification range $(20 \times-20,000 \times)$, continuous magnification times, simple specimen preparation, convenient orientation, etc., SEM moiré method is to be used to study the surface planar structure of three kinds of butterfly wings: Papilio maackii Menetries, Euploea midamus (Linnaeus), and Stichophthalma howqua (Westwood).

\section{Theoretical Analysis of Scanning Electron Microscopic Moiré}

\section{A. Formation Principle of Scanning Electron Microscopic Moiré}

SEM moiré is a group of fringe alternate with brightness and darkness, resulting from the interference between the specimen scanning image and SEM scanning lines. The specimen scanning image, or the periodic structure of specimen, is called specimen grating, while the pattern of SEM scanning lines is considered reference grating.

Suppose the reference grating pitch, i.e., the spacing between two adjacent SEM scanning lines, is $p_{r}$, the specimen grating pitch, i.e., the spacing between two adjacent lines of periodic structure on the specimen is $p_{s}$, and the included angle between the lines of reference grating and specimen grating is $\theta,-90^{\circ}$ $<\theta<90^{\circ}$. The spatial space frequencies are $f_{r}=$ $1 / p_{r}, f_{s}=1 / p_{s}$, respectively. To discuss conveniently, an orthogonal coordinate is set up here, setting one reference grating line as axis $x$, see Fig. 1.

Suppose the orders of reference grating and specimen grating lines are $m_{r}, m_{s}$, respectively, according to the two grating equations [22]:

$$
\begin{aligned}
& y=m_{r} p_{r}, \\
& y=\tan \theta x+\frac{m_{s} p_{s}}{\cos \theta} .
\end{aligned}
$$

Orders solutions of reference grating and specimen grating can be obtained

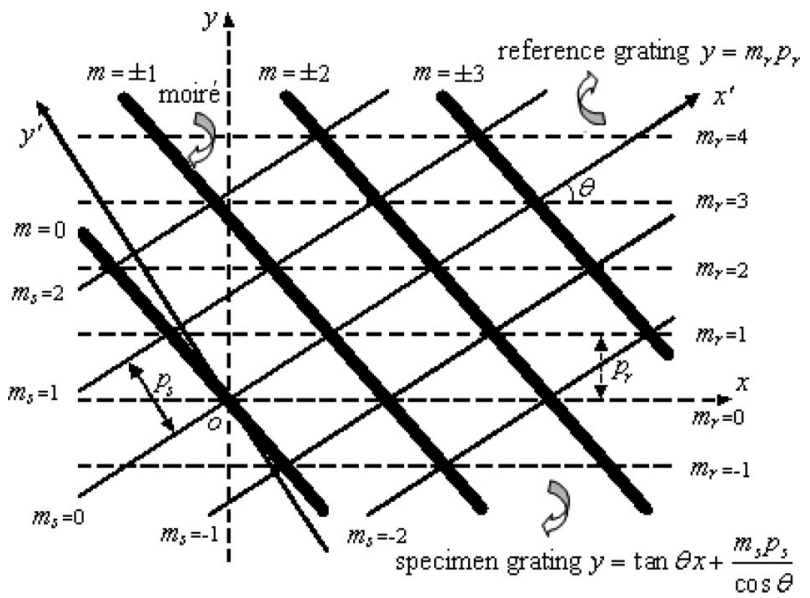

Fig. 1. Sketch of formation principle of SEM moiré.

$$
\begin{aligned}
& m_{r}=\frac{y}{p_{r}}, \\
& m_{s}=\frac{y \cos \theta-x \sin \theta}{p_{s}} .
\end{aligned}
$$

The transmissivity of reference grating is

$\zeta_{r}=1+\cos 2 \pi \frac{y}{p_{r}}=1+\cos 2 \pi f_{r} y=1+\cos 2 \pi m_{r}$.

Build a new coordinate system $x^{\prime} o y^{\prime}$, setting one specimen grating line as axis $x^{\prime}$. The transmissivity of specimen grating can be written as

$$
\zeta_{s}=1+\cos 2 \pi \frac{y^{\prime}}{p_{s}} .
$$

According to Eq. (4) and the coordinate transformation relation $y^{\prime}=y \cos \theta-x \sin \theta$, we can obtain

$$
\begin{aligned}
\zeta_{s} & =1+\cos 2 \pi \frac{y^{\prime}}{p_{s}}=1+\cos 2 \pi \frac{x \sin \theta+y \cos \theta}{p_{s}} \\
& =1+\cos 2 \pi m_{s} .
\end{aligned}
$$

Suppose a plane light beam with intensity of $\psi_{0}$ passes the two gratings continuously, the transmission intensity will be

$$
\begin{aligned}
\psi=\psi_{0} \zeta_{s} \zeta_{r}= & \psi_{0}\left(1+\cos 2 \pi m_{s}\right)\left(1+\cos 2 \pi m_{r}\right) \\
= & \psi_{0}\left(1+\cos 2 \pi m_{s}+\cos 2 \pi m_{r}\right. \\
& \left.+\cos 2 \pi m_{s} \cos 2 \pi m_{r}\right) \\
= & \psi_{0}\left[1+\cos 2 \pi m_{s}+\cos 2 \pi m_{r}\right. \\
& \left.+\frac{1}{2} \cos 2 \pi\left(m_{s}+m_{r}\right)+\frac{1}{2} \cos 2 \pi\left(m_{s}-m_{r}\right)\right] .
\end{aligned}
$$


The first item is the average light intensity; the second and the third items stand for reference grating and specimen grating, respectively; the fourth item stands for the denser moiré fringe whose frequency is the sum of the two gratings' frequencies; and the fifth item stands for the sparser moiré fringe whose frequency is the absolute value of the difference of the two gratings' frequencies. The former and latter fringes are called additive fringe and subtractive fringe, respectively. Since the additive fringe with higher frequency is too dense to be observed, only the subtractive fringe can be seen clearly. Therefore, the moiré fringe referred to commonly is the subtractive fringe. Suppose the order of moire fringe is $m$, the moiré order equation is

$$
m_{s}-m_{r}= \pm m \text {. }
$$

Although Eq. (9) stands for two expressions, it stands for only one group of fringe in fact, as fringe distributions are the same no matter if we adopt a positive sign or negative sign, since the transmissivity function is the cosine function $\zeta_{m}=1+$ $\cos 2 \pi m$. The positive and negative sign of $m$ is determined just by ordain, meaning that moiré fringe will be unique if the reference and specimen gratings are determined.

\section{B. Categories of Scanning Electron Microscopic Moiré}

SEM moiré includes parallel moiré and rotation moiré, while the latter consists of regular rotation moiré (parallel beelines distributed with equal spacing) and generalized rotation moiré (curves or beelines distributed with unequal spacing). Figures 2, 3, and 4 are formation sketch maps of SEM parallel moiré, regular rotation moiré, and generalized rotation moiré, respectively. Parallel moiré is composed of a group of parallel beelines distributed with equal spacing, which will appear when specimen grating and reference grating parallel and their frequencies match with each other. In the scanning process, a bright band appears when scanning lines are in the entity position, while a dark band appears when in the interspacing, see Fig. 2(c). One bright or dark band is referred to as a moiré fringe. When specimen grating has an angle with reference grating, there are two cases: if both the gratings are regular beeline gratings, the moiré, will be regular rotation moiré, composed of a group of parallel beelines, see Fig. 3(c); but if either is not a regular beeline grating or neither is, the formative moiré will be generalized rotation moiré, composed of a series of curves, or a group of

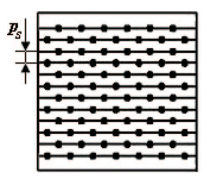

(a)

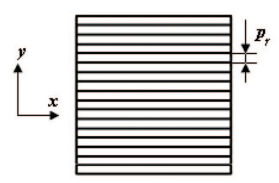

(b)

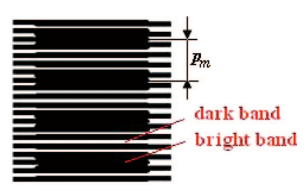

(c)
Fig. 2. (Color online) SEM parallel moiré. (a) Specimen surface structure, (b) SEM scanning lines, (c) SEM parallel moiré.

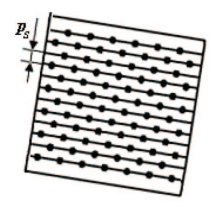

(a)

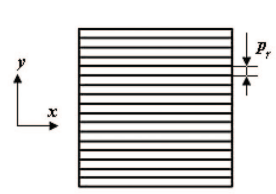

(b)

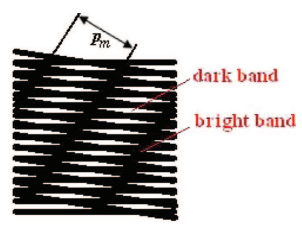

(c)
Fig. 3. (Color online) SEM regular rotation moiré. (a) Specimen surface structure, (b) SEM scanning lines, (c) SEM regular rotation moiré.

beelines distributed with unequal orientations or spacing, see Fig. 4(c).

\section{Analysis of Surface Structure of Specimen}

According to Eq. (9), if the shape of the reference grating is known, a correlative relation exists in the shape of specimen grating and moiré fringe. Since reference grating (SEM scanning lines) is composed of a group of horizontal beelines distributed with equal spacing, the shape of specimen grating (specimen surface structure) depends only on the shape of the moiré fringe. If the formative moiré is parallel moiré, the corresponding specimen grating is a group of horizontal beelines distributed with equal spacing; if regular rotation moiré, the specimen grating is a group of parallel beelines having an angle with SEM scanning lines and distributed with equal spacing; but if generalized rotation moiré, the specimen grating is composed of either a series of curves or a group of beelines with unequal orientations or spacing.

The results from multiple experiments show that moiré fringe patterns are distinct only when reference grating and specimen grating meet some matched conditions: the included angle between two gratings is small, and their frequencies or pitches match with each other; usually the frequency of one grating is $0.5-1.5$ times that of the other grating. It can be clearly seen that if distinct moiré fringe patterns appear, the included angle between the lines of specimen grating and SEM scanning lines is small, and the pitch of one is $0.5-1.5$ times that of the other. The direction of SEM scanning lines is usually horizontal, and the pitch is given by $p_{r}=L / N$, where $L$ is the length of scanning area and $N$ is the number of SEM scanning lines. To quantitatively analyze the planar character of specimen grating, the concept of virtual strain is imported. Suppose the specimen surface shape is obtained from in-plane deformation, and suppose its original shape before deformation is com-

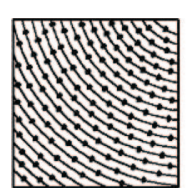

(a)

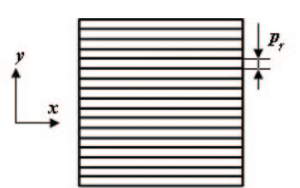

(b)

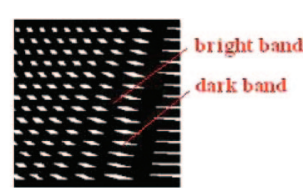

(c)
Fig. 4. (Color online) SEM generalized rotation moiré. (a) Specimen surface structure, (b) SEM scanning lines, (c) SEM generalized rotation moiré. 
pletely identical to reference grating (SEM scanning lines), the displacement and the corresponding strain are called virtual displacement and virtual strain, respectively.

The computing formula of virtual strain is the same as general strain in moiré method:

$$
\varepsilon_{y}=\frac{\partial v}{\partial y}=\frac{p_{r} \partial m}{\partial y}
$$

where $v$ is the virtual displacement in direction $y$ and moiré, fringe centerlines represent the contours of equal displacement; $p_{r}$ and $m$ have the same meaning as before, representing the reference grating pitch and the moiré fringe order, respectively.

To obtain the whole virtual strain field, it is not only necessary to assign moiré integral fringe orders but also to calculate the fractional fringe order through fringe order interpolation. The preferable interpolation is using segment-by-segment curve fitting interpolation algorithm, based on a continuous differential of the first derivative, i.e., second-order differential of displacement. The detailed processing is presented in [23].

Virtual strain field reflects the deformation of specimen grating relative to reference grating, which is applicable for all kinds of moiré. To explicitly describe the planar character of specimen grating, the spacing between two adjacent specimen grating lines in direction $y$ in full-field can be calculated through $p_{s y}$ $=p_{r}\left(1+\varepsilon_{y}\right)$ after virtual strain field is obtained. It is obvious that the positive and negative sign of virtual strain must be judged to determine $p_{s y}$ uniquely, which can be completed by observing the relationship of rotation directions between moiré fringe and specimen grating. If the rotation directions are the same, the corresponding virtual strain is compressive and negative sign should be adopted; else, if they are reversed, virtual strain is tensile and positive sign comes into use.

As moiré is highly sensitive to the planar shape of specimen grating and amplifies its deformation greatly, the error will shrink from moiré fringe processing to specimen grating characterizing.

\section{Experimental Results and Discussions}

The butterfly wings investigated in this paper are Papilio maackii Menetries, Euploea midamus (Linnaeus), and Stichophthalma howqua (Westwood) which were purchased in Xiangshan Park, Beijing, China. The instruments adopted are CSM950 SEM from Opton Corporation of Germany and Optelics C130 true color laser scanning confocal microscope (TCLSCM) made by Lasertec Corporation of Japan. TCLSCM was used to obtain the color images of butterfly wing as in the illumination of sunlight, using xenon with wavelength of 400-700 nm similar to visible light as laser source.

\section{A. Papilio Maackii Menetries}

Papilio maackii Menetries, one kind of Papilionidae, has black wings interspersed with brilliant golden green scales. It is known as the queen of the forest for its rich colors and graceful postures. The green part of one wing was chosen to be investigated in this experiment. Put the little part clipped from the whole wing under TCLSCM, and its true color image was obtained, see Fig. 5(a). The microstructures of the black scale and the green one, recorded by SEM, are shown in Figs. 5(b) and 5(c), respectively.

Among longitudinal ridges there are many microcross-ribs that seem not so much regular in this small region. Can these micro-cross-ribs form a grating in a large region, and which grating will they form? We will detect it through SEM moiré method.

After multiple adjustments, it is found that distinct moiré appears when the magnification of SEM is 400 and the number of horizontal scanning lines is 442 . The scanning area is $678 \times 586 \mu \mathrm{m}^{2}$, and the spacing between two adjacent scanning lines (reference grating pitch) is $p_{r}=586 / 442=1.326 \mu \mathrm{m}$. To determine the positive and negative sign of virtual strain, rotate the wing clockwise slightly to judge the relationship between the rotation directions of moire and the wing. Figures 5(d) and 5(e) are small parts with the size of $183 \times 255 \mu \mathrm{m}^{2}$, intercepted from the moiré fringe patterns recorded before and after rotation, respectively.

To analyze conveniently, serial numbers were given to each scale of butterfly wing. Scales of numbers 1 and 11 are green scales, with no micro-crossribs from Fig. 5(c) and thus no moiré fringes from Figs. 5(d) and 5(e). The detailed generation mechanism of the green color was reported in [1], and will not be discussed here. The black scales arouse our curiosity and interest us.

Take the scale of number 5 labeled in Figs. 5(d) and 5 (e) as an example to analyze. Through some treatment [24] such as filtering, binarization, moiré fringe thinning, and smoothness, thin moiré fringes before and after rotation can be obtained [Figs. 5(f) and $5(\mathrm{~g})]$. As the left moiré fringes on this scale rotated clockwise but the right moiré fringes rotated counterclockwise when the scale rotated clockwise, we know that the virtual strain is compressive in the left part but tensile in the right part, through the judgment principle of virtual strain we previously discussed: if the rotation directions are the same, the corresponding virtual strain is compressive; if they are reverse, virtual strain is tensile. Using moiré soft MOIRÉ ANALYSIS V0.92, virtual strain fields of the scale in the two states are given in Figs. 5(h) and 5(i), which show that there is both compressive and tensile virtual strain on the scale.

The change of the sign of virtual strain on the scale of number 5 can also be judged from the shape of the moiré fringes. It can be seen from Figs. 5(f) and $5(\mathrm{~g})$ that the right moiré fringes are left lower to right upper, but the left ones are right lower to left upper. In the middle there must be some moiré fringes with fractional orders, in one position of which the tangent is vertical. Since the calculated strain corresponding to vertical moiré fringes in field $V$ is zero, the sign of 

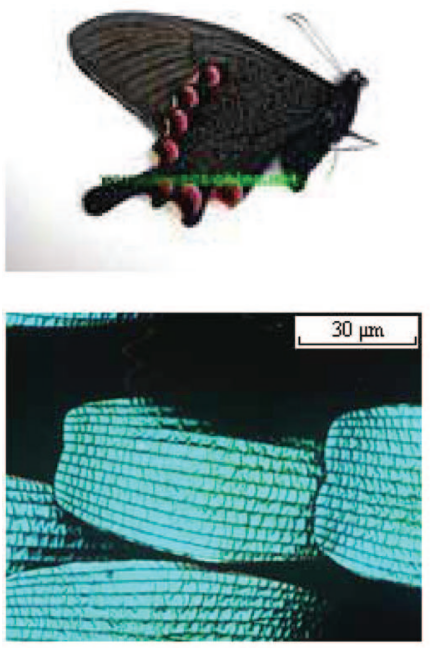

(a)

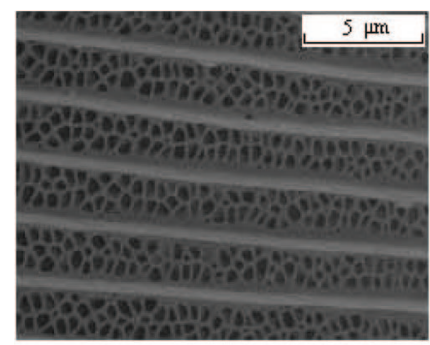

(b)

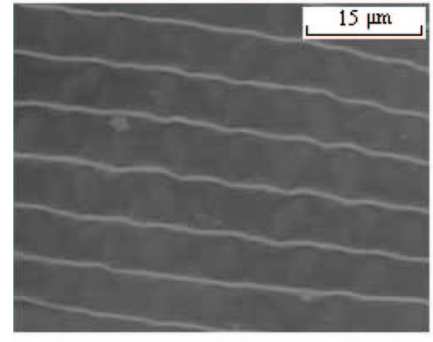

(c)

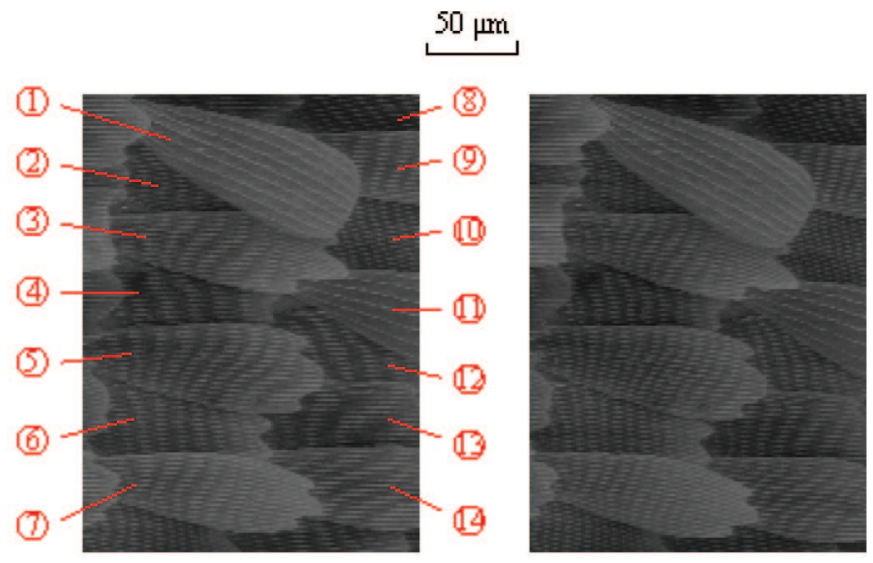

(d)

(e)
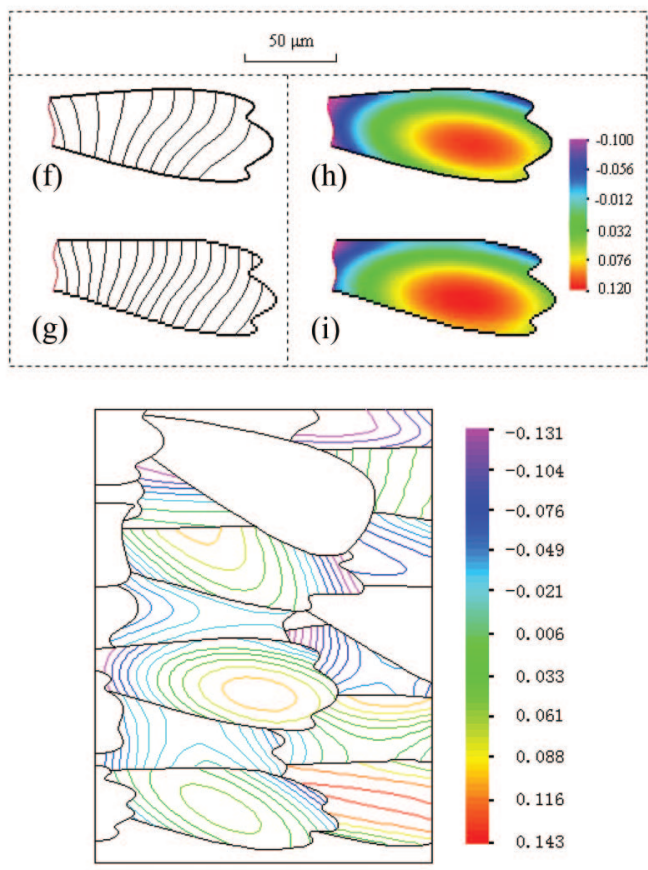

(j)

Fig. 5. (Color online) Papilio maackii Menetries (a) color image recorded by TCLSCM, (b) microstructure of black scale recorded by SEM, (c) microstructure of green scale recorded by SEM, (d) moiré fringe pattern before and (e) after clockwise rotation when SEM scanning lines $p_{r}=1.326 \mu \mathrm{m}$, (f) thin moiré fringes before, and (g) after clockwise rotation of scale of number 5 , (h) virtual strain fields of scale of number 5 before, and (i) after clockwise rotation relative to SEM scanning lines $p_{r}=1.326 \mu \mathrm{m}$, (j) full-field virtual strain fields of several scales before rotation relative to SEM scanning lines $p_{r}=1.326 \mu \mathrm{m}$.

strain must change from one side to the other side of vertical moiré fringes due to its continuity. As seen in Figs. 5(f) and 5(g), the tangents of moiré fringes in right-upper and left-middle regions are vertical; therefore, the virtual strain will change its sign in these regions, just like Figs. 5(h) and 5(i).

Since the reference grating pitch $p_{r}=1.326 \mu \mathrm{m}$ and the variation range of virtual strain is $\varepsilon_{y}=$ $0.100-0.120$, as seen in Figs. 5(h) and 5(i), the spacing between two adjacent specimen grating lines in direction $y$ can be obtained: $p_{s y}=p_{r}\left(1+\varepsilon_{y}\right)$ $=1.193-1.485 \mu \mathrm{m}$. According to the formation conditions of distinct moiré (the frequencies of two gratings match, and the included angle between them is small with $\cos \theta$ approaching 1), the normal spacing between two adjacent specimen grating lines $p_{s}=$ $p_{s y} \cos \theta$ is a little smaller than but approaches $p_{s y}$. That is to say, $p_{s}$ of the grating on the scale of this butterfly wing is a little smaller than 1.193$1.485 \mu \mathrm{m}$, which is approximately half of the normal spacing between two adjacent ridgelines (2.4-2.6 $\mu \mathrm{m})$. It means that the grating, generating SEM moiré through interferencing with SEM scanning lines, is formed by those micro-cross-ribs among longitudinal ridges, which may play an important role in its superhydrophobic characteristics as well as quick heat dissipation ability. The dimension and the characteristic of this grating can be acquired from Figs. 
5(h) and 5(i). Besides, moiré fringes on most scales are generalized rotation moiré fringes [Figs. 5(d) and $5(\mathrm{e})$, which shows that gratings on most scales are not regular gratings composed of beelines, but gratings composed of curves exhibiting some bend tendencies on the whole, which is just the natural state of butterfly wing in the Sun, and is very difficult to be observed under a high-power microscope.

To analyze the planar character of more scales of this butterfly wing in a larger range, full-field virtual strain, i.e., virtual strain of several scales, was calculated, adopting the same color code for convenient comparison; see Fig. 5(j). It can be seen that virtual strain of scales on the superlayer, such as scales of numbers $3,5,7,9,13$, and 14 , are almost tensile, except in some marginal area of some scales, such as scales of numbers $3,5,7$, and 13, while virtual strain of scales on the underlayer, such as scales of numbers $2,4,6,8,10$, and 12 , are almost compressive. It means that the spacing between two adjacent specimen grating lines in direction $y$ is larger on scales on the superlayer, except in some marginal area of some scales, and is smaller on scales on the underlayer. This particular structure distribution may contribute a lot in keeping the body temperature constant in the irradiation of intense sunlight through layer-by-layer diffraction of sunlight.

\section{B. Euploea Midamus (Linnaeus)}

Euploea midamus (Linnaeus) is of family Danaidae. The upper surface of its wings is brown, with a large shining purple-blue patch in the middle and several small white patches in the fringe and central area of each wing. The purple-blue part of one wing was chosen to be studied. Put the little part clipped from the whole wing under TCLSCM and we can see its true color image, see Fig. 6(a). The microstructure of one scale is in Fig. 6(b), which was recorded by SEM.

After multiple adjustments, it is found that distinct moiré appears when the magnification of SEM is 436 and the number of horizontal scanning lines is 442 . The scanning area is $620 \times 536 \mu \mathrm{m}^{2}$, and the reference grating pitch is $p_{r}=536 / 442=1.213 \mu \mathrm{m}$. Through rotating the wing clockwise slightly, it was found that moiré fringes rotated clockwise too, demonstrating that virtual strain is compressive. Here we only take one state (before rotation) as an example to analyze as in Fig. 6(c), which is a small part with the size of $217 \times 253 \mu \mathrm{m}^{2}$ intercepted from the whole image before rotation.

With the example of scale $*$ labeled in Fig. 6(c), through filtering, binarization, moiré fringe thinning, and smoothness, thin moiré fringes can be obtained [Fig. 6(d)]. Virtual strain field of the scale is given in
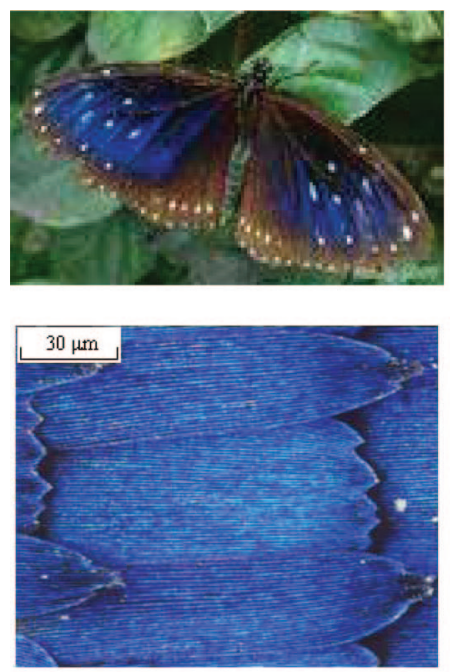

(a)

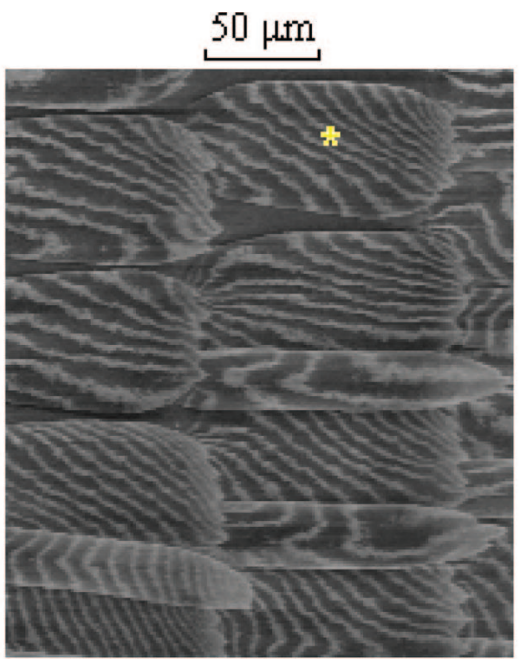

(c)

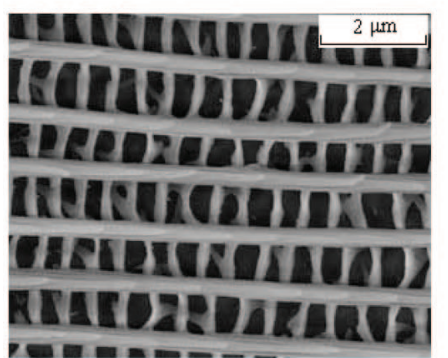

(b)

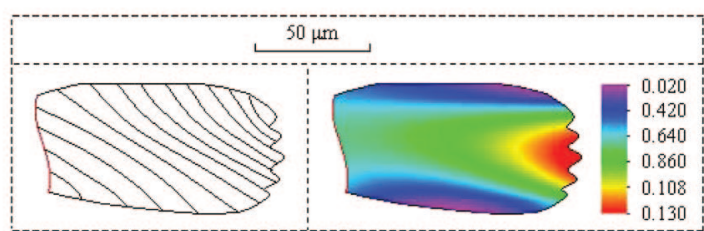

(d)

(e)

Fig. 6. (Color online) Euploea midamus (Linnaeus) (a) color image recorded by TCLSCM, (b) microstructure of scales recorded by SEM, (c) moiré fringe pattern when SEM scanning lines $p_{r}=1.213 \mu \mathrm{m}$, (d) thin moiré fringes of scale $*$, (e) virtual strain fields of scale $*$ relative to SEM scanning lines $p_{r}=1.213 \mu \mathrm{m}$. 
Fig. 6(e), showing that virtual strain on the scale is compressive.

Since $p_{r}=1.213 \mu \mathrm{m}$ and the variation range of virtual strain is $\varepsilon_{y}=-0.130$ to -0.020 , as seen in Fig. 6(e), the spacing between two adjacent specimen grating lines in direction $y$ can be obtained: $p_{s y}=$ $p_{r}\left(1+\varepsilon_{y}\right)=1.055-1.189 \mu \mathrm{m}$. According to the same analysis as butterfly Papilio maackii Menetries, we know that the normal pitch of the grating on the scale of the butterfly wing is a little smaller than 1.055-1.189 $\mu \mathrm{m}$. From Fig. 6(b), the longitudinal ridges can form a grating and the normal spacing between two adjacent ridgelines is $0.8-1.2 \mu \mathrm{m}$. It means the grating that forms moiré fringes with SEM scanning lines is just formed by the longitudinal ridges. What is more, moiré on most scales are also generalized rotation moiré [Fig. 6(c)], meaning that gratings on this kind of wing are also composed of a series of curves exhibiting some bend tendencies on the whole.

Since a single grating with a pitch of $0.8-1.2 \mu \mathrm{m}$ or frequency of 833-1250 lines $/ \mathrm{mm}$ will generate many colors in sunlight, while a group of gratings with different orientations can generate a single color in a large viewing angle, reported in [25,26], the phenomenon that this butterfly can exhibit purple-blue in a large viewing angle has a close relationship with those small gratings with different orientations on the butterfly wing, which can be clearly seen in moiré fringe pattern [Fig. 6(c)], where different moiré orientation means different grating orientation. The relationship between different orientations of gratings on different scales can be characterized by the different shape of moiré fringes.

\section{Thaumantis Diores (Doubleday)}

Thaumantis diores (Doubleday) belongs to Amathusiidae. The upper surface of wings is dark brown with a prominent large shining metallic blue patch in the middle of each wing. The blue part of one wing was chosen for study, and the true color image of a little part clipped from this blue part was recorded by TCLSCM [Fig. 7(a)], showing that the blue part contains both blue and brown scales. The microstructures of blue and dark brown scales of the blue part are in Figs. 7(b) and 7(c), respectively, both of which were recorded by SEM.

As to the blue part, it is found that distinct moiré appears when the magnification of SEM is 662 and the number of horizontal scanning lines is 442 after multiple adjustments. The scanning area is

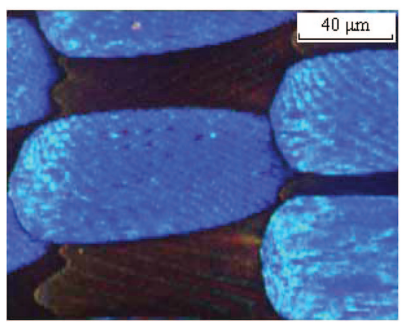

(a)

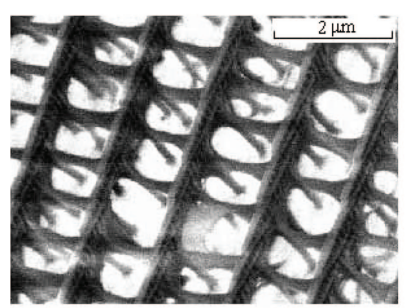

(b)

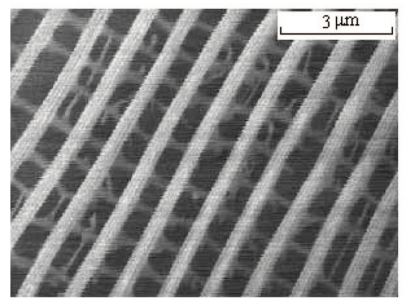

(c)
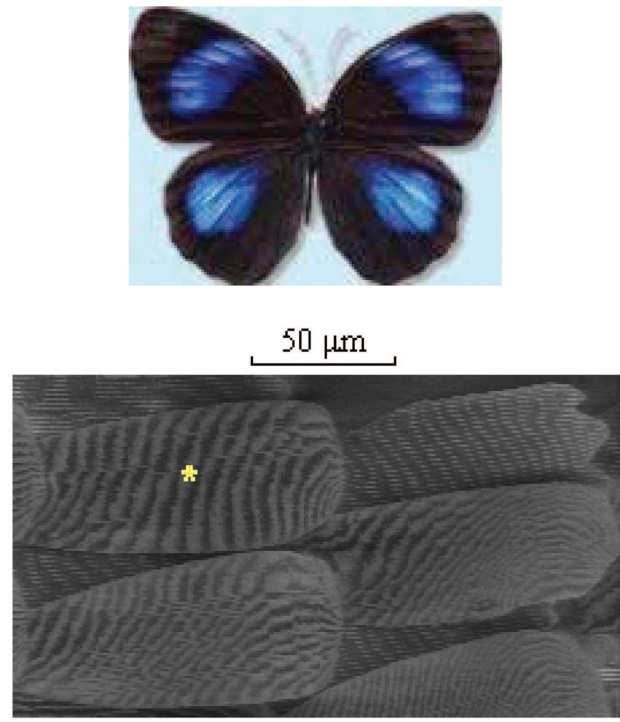

(d)

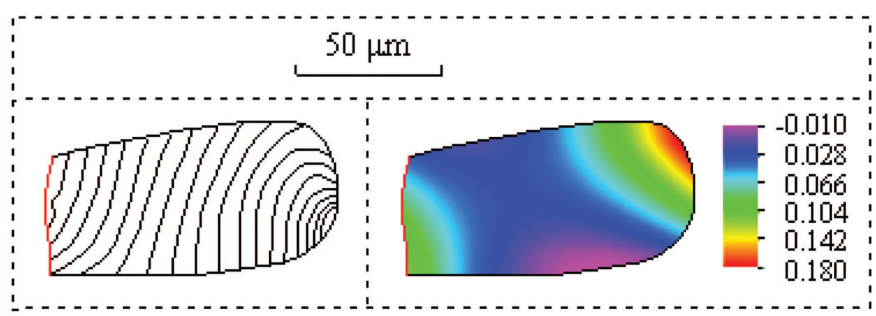

(e)

(f)

Fig. 7. (Color online) Thaumantis diores (Doubleday) (a) color image recorded by TCLSCM, (b) microstructure of blue scales recorded by SEM, (c) microstructure of dark brown scales recorded by SEM, (d) moiré fringe pattern when SEM scanning lines $p_{r}=0.801 \mu \mathrm{m}$, (e) thin moiré fringes of scale $*$, (f) virtual strain fields of scale $*$ relative to SEM scanning lines $p_{r}=0.801 \mu \mathrm{m}$. 
$410 \times 354 \mu \mathrm{m}^{2}$, and the spacing between two adjacent scanning lines (reference grating pitch) is $p_{r}=$ $354 / 442=0.801 \mu \mathrm{m}$. Here also we only take one state (before rotation) as an example to analyze as in Fig. 7(d), which is a small part with the size of $217 \times 253 \mu \mathrm{m}^{2}$ intercepted from the whole image before rotation.

With the example of scale * labeled in Fig. 7(d), thin moiré fringes can be obtained through filtering, binarization, moiré fringe thinning, and smoothness, see Fig. 7(e). Since moiré fringes in the right-lower region rotated clockwise but those in the other regions rotated counter-clockwise when the scale rotated clockwise, we know that virtual strain is compressive in the right-lower region but tensile in the other regions. Virtual strain field of this scale is given in Fig. 7(f), showing that there are both compressive and tensile virtual strain on this scale, which can also be judged from the phenomenon that moiré fringes deflect in right-lower region.

Since $p_{r}=0.801 \mu \mathrm{m}$ and the variation range of virtual strain is $\varepsilon_{y}=0.010$ to -0.180 , as seen in Fig. $7(\mathrm{f})$, the spacing between two adjacent specimen grating lines in direction $y$ is given by $p_{s y}=p_{r}\left(1+\varepsilon_{y}\right)$ $=0.657-0.809 \mu \mathrm{m}$. We also know that the normal pitch of the grating on the scale of this butterfly wing is a little smaller than $0.657-0.809 \mu \mathrm{m}$. In addition, moiré fringes on most scales are generalized rotation moiré fringes too [Fig. 7(d)], meaning that gratings on the wing of butterfly Thaumantis diores (Doubleday) are also composed of a series of curves exhibiting some bend tendencies on the whole.

From Fig. 7(b), the longitudinal ridges can form a grating and the normal spacing between two adjacent ridgelines is $1.3-1.6 \mu \mathrm{m}$, almost two times as calculated results $0.657-0.809 \mu \mathrm{m}$. This is rather similar to butterfly Papilio maackii Menetries, which also shows that gratings generating moiré fringes are formed by micro-cross-ribs among ridges. What is more, it is obvious that there are moiré fringes on all scales through Figs. 7(a)-7(d), meaning that gratings with similar pitch exist on both blue and dark brown scales on the blue part of the wing. Then why are some scales blue but others dark brown? It can be clearly seen from Fig. 7(a) that there are many pigments on the blue scales but nothing on the dark brown scales. Pigments can strongly modulate the spectral distribution of the scattered and/or reflected light when the absorption of these substances is only substantial in a restricted wavelength range $[27,28]$. Accordingly, the blue color arises from the interaction of pigments and its special structure, distinguished as either physical or chemical colors.

The difference in the structure between blue scales carrying pigments and dark brown scales carrying nothing is shown in Figs. 7(b) and 7(c). Then we can come to a conclusion that blue scales with gratings and pigments contribute to the blue color of the wing, while dark brown scales with only gratings play an important role in its quick heat dissipation ability to keep the body temperature constant in the irradia- tion of intense sunlight. This special configuration is a marvelous characteristic of this kind of butterfly wing and a magic wonder of the nature, which will inspire humans greatly in bionic design in many fields.

\section{Conclusions}

(1) SEM moiré method is a simple, nonlocal, economical, and effective technique for determining which grating exists, measuring both the dimension and the whole structural character of the grating on each scale, as well as characterizing the relationship between gratings on different scales of one butterfly wing. Therefore, moiré method is a useful tool to assist with characterizing the structure of butterfly wings and explaining their excellent properties.

(2) Moiré fringes on most scales on all kinds of butterfly wings are generalized rotation moiré fringes, which shows that gratings on most scales are not regular gratings composed of beelines, but gratings composed of curves exhibiting some bend tendencies on the whole, which is just the natural state of butterfly wing in the sunlight, and is very difficult to be observed under high-power microscopy only.

(3) For the golden green part of Papilio maackii Menetries, micro-cross-ribs among longitudinal ridges on brown scales can form a grating with $p_{s}$ a little smaller than 1.193-1.485 $\mu \mathrm{m}$, which may play an important role in its superhydrophobic characteristics as well as quick heat dissipation ability. From the calculated strain field, the spacing between two adjacent specimen grating lines in direction $y$ is larger on scales on the superlayer, except in some marginal area of some scales, but is smaller on scales on the underlayer. This particular structure distribution may contribute a lot in keeping the body temperature constant in the irradiation of intense sunlight through layer-by-layer diffraction of sunlight.

(4) For the purple-blue parts of Euploea midamus (Linnaeus), the grating generating moiré fringes through interferencing with SEM scanning lines is just formed by longitudinal ridges. Gratings with pitch of $0.8-1.2 \mu \mathrm{m}$ and with different orientations on all scales contribute a lot in generating purpleblue in a large viewing angle. The relationship between different orientations of gratings on different scales can be characterized by the different shape of SEM moiré fringes.

(5) For the blue parts of Thaumantis diores (Doubleday), gratings with similar pitch of approximately $0.657-0.809 \mu \mathrm{m}$ exist on both blue and dark brown scales on the blue part of the wing. Their whole planar characteristics can be described by SEM moiré fringes. Blue scales with gratings and pigments contribute to the blue color of the wing, while dark brown scales with only gratings may play an important role in its quick heat dissipation ability to keep the body temperature constant in the irradiation of intense sunlight.

The work is supported by the National Basic Research Program of China through grant 
2004CB619304, the National Natural Science Foundation of China (under grants 10625209, 10121202, and 10472050), the Excellent Young Teacher Program of the Chinese Ministry of Education, and the Program for New Century Excellent Talents (NCET) in University, Ministry of Education. The authors also gratefully acknowledge the financial support of Beijing Natural Sciences Foundation through grant 3072007 and the opening funds from LNM, Chinese Academy of Science.

\section{References}

1. P. Vukusic, J. R. Sambles, and C. R. Lawrence, "Colour mixing in wing scales of a butterfly," Nature 404, 457 (2000).

2. P. Vukusic and J. R. Sambles, "Shedding light on butterfly wings," Proc. SPIE 4438, 85-95 (2001).

3. C. Lawrence, P. Vukusic, and J. R. Sambles, "Grazingincidence iridescence from a butterfly wing," Appl. Opt. 41, 437-441 (2002).

4. B. Gralak, G. Tayeb, and S. Enoch, "Morpho butterflies wings color modeled with lamellar grating theory," Opt. Express 9, 567-578 (2001).

5. J. B. Harris, T. W. Preist, J. R. Sambles, R. N. Thorpe, and R. A. Watts, "Optical response of bigratings," J. Opt. Soc. Am. A 13, 2041-2049 (1996).

6. S. A. Khan, D. N. Qu, and R. E. Burge, "Experimental analysis of diffraction by wavelength-sized metallic gratings in the microwave region," Opt. Eng. 32, 3249-3253 (1993).

7. H. Ghiradella, D. Aneshansley, T. Eisner, R. Silberglied, and H. E. Hinton, "Ultraviolet reflection of a male butterfly: interference color caused by thin-layer elaboration of wing scales," Science 178, 1214-1217 (1972).

8. P. Vukusic, J. R. Sambles, C. R. Lawrence, and R. J. Wootton, "Quantified interference and diffraction in single Morpho butterfly scales," Proc. R. Soc. London Ser. B 266, 1403-1411 (1999).

9. J. L. Brink and M. E. Lee, "Confined blue iridescence by a diffracting microstructure: an optical investigation of the Cynandra opis butterfly," Appl. Opt. 38, 5282-5289 (1999).

10. R. B. Morris, "Iridescence from diffraction structures in the wing scales of Callophrys rubi, the Green Hairstreak," J. Entomol. Ser. A 49, 149-154 (1975).

11. D. G. Stavenga, M. A. Giraldol, and B. J. Hoenders, "Reflectance and transmittance of light scattering scales stacked on the wings of pierid butterflies," Opt. Express 14, 4880-4890 (2006).

12. L. P. Biro, "Role of photonic-crystal-type structures in the ther- mal regulation of a Lycaenid butterfly sister species pair," Phys. Rev. E 67, 021907 (2003).

13. Q. Cong, G. H. Chen, Y. Fang, and R. Luquan, "Superhydrophobic characteristics of butterfly wing surface," J. Bioeng. 1, 249-255 (2004)

14. Y. M. Xing, F. L. Dai, and W. Yang, "Experimental study about nano-deformation field near quasi-cleavage crack tip," Sci. China Ser. A: Math. Phys., Astron. 43, 963-968 (2000).

15. R. Weller and B. M. Shepherd, "Displacement measurement by mechanical interferometry,” Proc. Soc. Exp. Stress Anal. 6, 35-38 (1948).

16. B. Han, D. Post, and P. Ifju, "Moiré interferometry for engineering mechanics: current practices and future developments," J. Strain Anal. Eng. Des. 36, 101-117 (2001).

17. S. Kishimoto, M. Egashira, and N. Shinya, "Micro-creep deformation measurement by a moiré method using electron beam lithography and electron beam scan,” Opt. Eng. 32, 522-526 (1993).

18. H. Chen and D. Liu, "Advances in scanning electron microscope moire," Exp. Mech. 41, 165-173 (2001).

19. H. M. Xie, S. Kishimoto, and A. Asundi, "In-plane deformation measurement using the atomic force microscope moiré method," Nanotechnology 11, 24-29 (2000).

20. C. M. Liu and L. W. Chen, "Digital phase-shifting atomic force microscope moiré method," J. Phys. D 38, 1182-1189 (2005).

21. B. Pan, H. Xie, S. Kishimoto, and Y. Xing, "Experimental study of moiré method in laser scanning confocal microscopy," Rev. Sci. Instrum. 77, 043101 (2006).

22. Y. Meiwen, Optical Holography and Information Processing (Academic, 1983), pp. 193-195.

23. Z. Wang, "Development and application of computer-aided fringe analysis," Ph.D. dissertation (U. Maryland at College Park, 2003).

24. W. Qiu, Y. L. Kang, Q. H. Qin, and W. T. Li, "Regional identification, partition, and integral phase unwrapping method for processing moiré interferometry images," Appl. Opt. 45, 6551-6559 (2006).

25. T.-H. Wong and M. C. Gupta, "Color generation in butterfly wings and fabrication of such structures," Opt. Lett. 28, 23422344 (2003).

26. R. B. Hwang and S. T. Peng, "Performance evaluation of a bigrating as a beam splitter," Appl. Opt. 36, 2011-2018 (1997).

27. H. Descimon, "Biology of pigmentation in Pieridae butterflies," in Chemistry and Biology of Pteridines, W. Pfleiderer, ed. (De Gruyter, Berlin, 1975), pp. 805-840.

28. J. M. Janssen, A. Monteiro, and P. M. Brakefield, "Correlations between scale structure and pigmentation in butterfly wings," Evol. Dev. 3, 415-423 (2001). 